ISSN: $1130-3743$

DOI: http://dx.doi.org/10.14201/teoredu2014261115140

\title{
EDUCACIÓN AMBIENTAL, INTELIGENCIA ESPIRITUAL Y NATURALEZA
}

\author{
Environmental Education, Spiritual Intelligence \\ and Nature
}

\section{Éducation à l'environnement, l'intelligence spirituelle et nature}

Jordi Puig Baguer*, Fernando Echarri IrIBARren** y María Casas Jericó**** Universidad de Navarra. Facultad de Ciencias. Departamento de Biología Ambiental.31080 Pamplona. "ipbaguer@unav.es. * feiribarren@alumni.unav.es. ***: mcasas.1@alumni.unav.es

Fecha de recepción: marzo de 2014

Fecha de aceptación: junio de 2014

Biblid [(1130-3743) 26, 2-2014, 115-140]

\section{RESUMEN}

La conciencia ambiental es característica en nuestra cultura, y plantea múltiples retos educativos. Las elecciones ambientales no dejan de construirnos... o destruirnos, tanto personal como colectivamente, en dimensiones corporales o culturales, en el individuo humano y en su paisaje. Entre ser humano y naturaleza existe un vínculo, común a ambos, de humanidad y naturalidad que no puede olvidarse en la educación sin erosionar el valor y el respeto del ser humano y la naturaleza a la vez.

Este artículo busca impulsar la educación en medio ambiente mediante el desarrollo de la inteligencia espiritual y viceversa, con el fin de alimentar nuestro conocimiento con una profundidad y universalidad que necesitamos. En particular, destaca el papel cultural de la ciencia ecológica y resalta que la diferencia esencial 
entre los seres humanos y su medio natural permite $-\mathrm{y}$ debe- alimentar el respeto de ambos a través de la importancia y valor del recíproco.

Palabras clave: medio natural; naturaleza y cultura; ética ambiental; pertenencia ambiental; ecología; ciencias ambientales; humanidades y medio ambiente.

\section{SUMMARY}

Our culture has developed an environmental awareness that has to meet many educative challenges. Environmental choices may build us up... or wreck us, individually and collectively, damaging both our bodies and cultures, even our landscapes. There is a shared link between human beings and nature, made out of both humanity and naturalness. The value and respect for both human beings and nature are damaged to the same extent that this link falls into oblivion in education.

This paper seeks to foster environmental education through spiritual intelligence... and vice versa. This strategy aims at nourishing our environmental stance with depth and universality, in order to reach the culture that lies beyond the materiality of the environment. The role of the science of ecology is stressed. The essential distinction between human beings and their natural environment is seen as a call to nourish their respective values, which depend in each other.

Key words: natural environment; nature and culture; environmental ethics; ecology; environmental sciences; environmental humanities.

\section{SOMMAIRE}

Dans notre culture on a développé une sensibilisation à l'environnement, et cela pose de nombreux défis éducatifs. Les choix environnementaux n'arrêtent pas de nous construire... ou de nous détruire, aussi bien individuellement que collectivement, dans des dimensions corporelles ou culturelles, dans l'individu en temps qu'humain et dans son paysage. Entre l'homme et la nature, il existe un lien, commun, d'humanité et de naturel que l'on ne peut pas oublier en ce qui concerne l'éducation, sans endommager la valeur et le respect des humains et de la nature en même temps.

Cet article cherche à stimuler l'éducation de l'environnement par l'intermédiaire du développement de l'intelligence spirituelle et vice versa, afin de nourrir notre connaissance avec une profondeur et l'universalité d'ont on a besoin. En particulier, le rôle culturel de la science écologique est mis en évidence et il en ressort que la différence essentielle entre les humains et leur environnement naturel permet -et doit- nourrir le respect des deux, par l'intermédiaire de l'importance et la valeur du réciproque.

Mots clés: environnement; nature et culture; l'éthique environnementale; l'environnement appartenant; écologie; sciences de l'environnement; les sciences humaines et l'environnement. 


\section{INTRODUCCIÓN Y TESIS DEFENDIDA EN EL ARTÍCULO}

La conciencia ambiental es una tendencia emergente de nuestra cultura y plantea un reto específico a la educación. Desde la psicología ambiental se ha progresado en el conocimiento de los mecanismos del comportamiento en esta materia. Se persigue mejorar la conducta ambiental (Kaiser, 2014), por ejemplo, actuando sobre el entorno humano (Gärling, 2014), el contacto con la naturaleza (Olivos-Jara, Aragonés y Navarro-Carrascal, 2013), la motivación al compromiso (Baca-Motes, Brown, Gneezy, Keenan y Nelson, 2013), buscando un empleo políticamente eficaz del conocimiento científico (Ernst y Wenzel, 2014) o articulándola con la educación en edades específicas (Kerret y Ronen, 2014). Este artículo busca reflexionar sobre la educación ambiental y la inteligencia espiritual, y proponer y argumentar algunas ideas que permitan articular propuestas educativas. Se introduce muy brevemente una propuesta, cerrando el artículo, que busca simplemente apuntar una de sus posibles materializaciones en la educación ambiental, sin desarrollarla.

La tesis que defiende este artículo es que la inteligencia espiritual y el aprendizaje de cómo entendernos y comportarnos en el medio ambiente natural (y en relación con él) deberían ir más de la mano en la educación, por ejemplo, en temas como el consumo (Koh y Lee, 2012). La relación del ser humano con el mundo natural puede (y debería...) aportar un alimento extraordinario no sólo al cuerpo, sino también al espíritu humano. El cual, a su vez, puede (y debería...) conducirnos a una vida cuyas acciones estuvieran integradas en nuestro medio, de modo que no lo devaluaran: en equilibrio con él, respetuosa con su modo de ser material, y plena de sentido natural y humano. La atención a la inteligencia espiritual permite llegar a raíces profundas, a veces difíciles de apreciar, de los hábitos que construyen los problemas ambientales. En palabras de Aldo Leopold, a quien se citará en más ocasiones por la notoriedad que su propuesta ética ha ido adquiriendo, hasta convertirle en un clásico, "...que la tierra debe ser amada y respetada es una extensión de la ética. Que la tierra produce una cosecha cultural es un hecho conocido desde hace tiempo, pero que es olvidado con frecuencia últimamente" (Leopold, 1949, Foreword [x] $)^{1}$.

En adelante se usarán de forma similar o análoga una colección de términos emparentados: naturaleza, tierra, medio ambiente, medio natural, medio ambiente natural, entorno, ecosistema... En contextos disciplinarios específicos estos términos merecerían cuidadosas distinciones o, al menos, aclaraciones. Se ha optado por usarlos de modo flexible, en ocasiones intercambiable, buscando una mayor fluidez narrativa, y la familiaridad con el lenguaje educativo del amplio entorno social en el que debe integrarse la educación ambiental. Si la exigencia de exactitud científica apremia por un lado, la flexibilidad en el uso de esas expresiones

1. Las citas textuales de originales en inglés han sido traducidas por los autores. 
facilita que la atención del lector se dirija más fluidamente hacia las existencias no artificiales que salen al encuentro de la mujer y del hombre, contundentes con su materialidad, y que van más allá de la del cuerpo humano: el universo y la Tierra; montañas y mares; la atmósfera, el clima y el agua; las costas, planicies y valles, con sus tierras más o menos fértiles; los bosques, matorrales y praderas... Los ecosistemas todos y sus componentes relacionados, con sus hábitats, plantas y animales.

En este trabajo, por último, la espiritualidad se entiende como un rasgo propio de todo ser humano. Se sigue así un modo de abordarlo específico, adoptado por otros trabajos en el ámbito de la educación (Jennings, 2008; Benson y Roehlkepartain, 2008). Lo espiritual puede abrirse a enfoques religiosos específicos. Sin embargo, la propuesta educativa que se presenta deja a la elección de los lectores la vía a tomar. Se busca así un punto de encuentro o de partida válido para la variedad de creyentes (por ejemplo, en una divinidad creadora) o no creyentes, sin entrar en más distinciones, que no son el objeto de trabajo. Ni se exige entrar a la espiritualidad desde enfoques vinculados a las religiones o creencias... ni se les cierra el paso, pues también pueden contribuir eficazmente al respeto que se debe a la naturaleza. Se busca así abrirse a la variedad de quienes se afanan en la educación ambiental y procurar superar desencuentros en esta cuestión sin pretender resolverlos (ni se ha intentado en este artículo ni ven sus autores que deban hacerlo). Sí se busca contribuir en lo posible a ampliar las alianzas en el cuidado conjunto de la naturaleza y el espíritu humano.

¿Y por qué se ha escogido el enfoque espiritual, si se presta a una confusión no deseada con opciones religiosas específicas? Porque el acercamiento espiritual dota a la mirada humana de una profundidad y universalidad muy necesarias. En particular, en la educación relativa al papel de lo ambiental en la vida humana, un aspecto relevante para la vida cotidiana de todas las culturas y profesiones, a todas las edades y quehaceres, y de atención obligatoria para buscar una convivencia sostenible en el seno de las sociedades y comunidades (Schelly, Cross, Franzen, Hall y Reeve, 2012), y entre ellas (Root, 2010). Lo sugiere la misma definición de inteligencia espiritual que se ha escogido: "... nuestra capacidad humana de plantear preguntas fundamentales sobre el sentido de la vida; y de experimentar simultáneamente la perfecta conexión entre cada uno de nosotros y el mundo en el que vivimos" (Wolman, 2001, 1).

\section{EDUCACIÓN AMBIENTAL E INTELIGENCIA ESPIRITUAL: ANTECEDENTES SELECCIONADOS}

Se han seleccionado algunas publicaciones que abordan directa o indirectamente la educación ambiental y la inteligencia espiritual, para ofrecer un marco suficiente en el que proponer con sentido de continuidad los contenidos y argumentaciones que siguen a este apartado. En el contexto de la teoría de las inteligencias múltiples, se ha propuesto que la educación es lo más efectiva posible cuando es interdisciplinar y multicultural, porque la mente humana engloba numerosas formas de inteligencia, cada una de las cuales aprende de un modo distinto (Caduto, 
1998). A las siete inteligencias originalmente descritas por la teoría (lingüística, musical, lógico-matemática, espacial, corporal-cenestésica, intrapersonal e interpersonal), Gardner (1983, 2000) añadió más tardíamente la inteligencia naturalista, que consistiría en la "capacidad humana de discernir entre seres vivos (plantas y animales) y la sensibilidad hacia otras características del mundo natural (nubes, configuraciones rocosas)" (Caduto, 1998, 12). Por su parte, Caduto propuso añadir cuatro inteligencias a la lista anterior (ambiental, emocional, intuitiva y espiritual). Entre ellas, dos se refieren a los temas centrales que se abordan en este artículo. Caduto diferenció su propuesta de inteligencia ambiental, de la naturalista de Gardner. La inteligencia ambiental abarcaría "todos los aspectos de nuestra comprensión y conciencia de la tierra, así como nuestra capacidad para mantener una relación saludable y sostenible con el mundo natural" (Caduto, 1998). Su trabajo en torno a su educación ecológica busca promover el concurso de las múltiples inteligencias en la educación, incluida la espiritual. El alcance de sus propuestas educativas se puede reflejar con estas palabras:

La educación ecológica [...] promueve la comprensión y la conectividad ecológicas, la conciencia y apreciación ambiental, así como la custodia de la tierra. Es una forma de enseñar apropiada para representar el carácter interconectado e interdependiente de nuestra casa tierra. [...] Muy a pesar nuestro, la aplicación del conocimiento científico sin la orientación de la sabiduría ha conducido a la forma de existencia de hoy en día, desequilibrada e insostenible. [...] La educación ecológica implica temas morales y valores porque enseña acerca de la vida, acerca de relaciones humanas y acerca de interacciones entre la gente y el medio ambiente (Caduto, 1998, 12-13).

En otros ámbitos, y mediante entrevistas a amantes de la naturaleza, se ha tratado de comprender la dimensión espiritual que encierra su experiencia; se ha explorado cómo el verla imbuida de significado, valor intrínseco o sacralidad parece generar un mayor sentido de responsabilidad ambiental, por lo que se ha afirmado que el encuentro entre espiritualidad y naturaleza es de importancia creciente para el desarrollo sostenible (Hedlund-de Witt, 2013).

Buscando superar las limitaciones de enfoques exclusivamente prácticos o económicos, se ha propuesto que lo ambiental que llamamos genéricamente "verde" es un valor, que debe ser preservado por sí mismo, no por enriquecernos económicamente, sino porque desde siempre ha enriquecido la vida, en un sentido más pleno que el económico:

Al fin y al cabo, de eso es de lo que trata también una ética de la conservación, que declara que mantener nuestro mundo natural es un valor que es imposible cuantificar, pero también ignorar, a causa de la pura belleza, asombro, alegría y magia que la naturaleza trae al hecho de estar vivo (Friedman, 2008, 314).

Se ha estudiado también cómo superar la fragmentación de los currículos educativos, que reflejaría una visión del mundo igualmente fragmentada y reductiva, 
que fomentaría la alteración del equilibrio natural, hacia la destrucción: "Profesores y estudiantes se sienten seguros en un currículo minimalista en el cual las materias y los departamentos se encuentran aislados, donde el conocimiento se separa del que aprende, donde se busca el significado prescindiendo del contexto, donde el aprendizaje es juzgado en relación a la memorización, y donde se inmortaliza la competencia" (Slabbert y Hattingh, 2006, 702). En torno a todas estas cuestiones, y dándoles continuidad, se estima oportuno proponer las reflexiones que siguen.

\section{CONTENIDOS Y ARGUMENTACIONES DE LA TESIS PROPUESTA}

\subsection{Sobre los tipos de vínculos ambientales, su alcance material o espiritual, y cómo pueden condicionar las elecciones}

La educación ambiental lleva décadas fomentando la responsabilidad y el respeto hacia el mundo natural en el que vivimos y del que somos parte, mediante innumerables vías de relación en el seno de una comunidad de vida que nos liga inseparablemente a los demás, por más que el papel del ser humano en ella haya cambiado a lo largo de la historia (Hughes, 2001). Entre otros muchos aprendizajes, y partiendo de esta pertenencia a un medio natural y social en interacción, esta disciplina intenta lograr que se valore más el ser-ser en un ambiente, y junto con él y los demás- que el mero tener -un verbo que, frente al verbo ser, limita muy significativamente las posibilidades de relación con el entorno y con los demás (Hamilton, 2010)-. Pero, a este respecto, la educación no siempre consigue transmitir adecuadamente dos aspectos simultáneos de la relación del ser humano con el medio ambiente, y en su seno. De una parte, la profundidad -y aquí se encuentra un primer valor- con la que estamos anclados necesariamente al medio ambiente natural. Nos la desvelan las reglas, el modo de ser y de funcionar de lo natural que la ciencia experimental va descubriendo -no podemos, por ejemplo, dejar de alimentarnos o de tomar líquidos sin sufrir daño-. De otra, la posibilidad alternativa de relacionarnos con ese medio de un modo libre y también profundo -sí podemos escoger lo que comemos y bebemos, o contaminar el agua... y también depurarla; e incluso escribir su poesía o expresarla musicalmente-. Es éste un modo escogido de relación o, al menos, mudable: al alcance de la elección. Y de la educación.

Estamos inevitablemente en relación con nuestro medio ambiente natural. Lo manifiesta constantemente el desarrollo de la ecología, que ha impulsado -entre tantas otras- las nociones de organización en sistemas y de ecodependencia (Barton, 2006; Morin, 2006). Lo pueden recordar experiencias tan cotidianas como la alimentación, la higiene o la búsqueda de refugio frente a la intemperie para cuidar la salud y el bienestar. Pero el modo concreto de satisfacer estas necesidades materiales, con todo lo que pueda provocar en el medio (agricultura, pesca, urbanización, consumo energético, transporte...), no está escrito forzosamente de antemano del modo específico en que se ha hecho realidad territorial e histórica. Se 
podría haber actuado de otro modo. Y en muchas ocasiones, era un deber. De ahí, por ejemplo, el interés en buscar alternativas de gran alcance cultural, contando con la educación (Hart, 2010).

En cualquier caso, la condición corpórea del ser humano obliga a interactuar con el medio; y entre quienes lo habitan, a través de él. Un medio que tiene unas reglas -científicas- que no se inventan: se descubren. Pero más allá de ellas, y de las necesidades impuestas por tener un cuerpo, queda margen de libertad para las elecciones ambientales. Sean más o menos libres o conscientes, o implícitamente y tal vez inadvertidamente vividas a través del uso y consumo concretos, estas elecciones afectan inevitablemente a la totalidad del ser humano. No sólo a lo que llamamos corporal. "Quien no posee una granja se enfrenta a dos peligros espirituales [entendidos como lo que es distinto a lo físico o material]. El de suponer, en primer lugar, que el desayuno procede de la tienda de comestibles, y en segundo, que el calor procede de la calefacción" (Leopold, 1949, 6)².

Toda decisión libre puede ser tan acertada como estar amenazada de contradicción o fracaso. La relación del ser humano con el medio, y en su seno, puede llegar a ser muy valiosa. O lo contrario, como demuestran tantos contextos urbanos insalubres (Galea y Vlahov, 2005) o los impactos que el modo de vida urbano genera (Newman, 2006). Además, la falta de naturalidad va con frecuencia acompañada de la falta de justicia (Taylor, 2010). Sea en los barrios insalubres o en cualquier lugar físico o cultural donde la naturalidad es combatida por una cultura que debería, en cambio, integrarla.

La comprensión del modo de ser y del valor de la naturaleza puede promover actitudes de admiración y respeto, que faciliten una interacción humana con lo natural sin necesidad de consumirlo irresponsablemente ni, en ocasiones, de alterarlo: "Para una minoría de nosotros, la oportunidad de ver gansos es más importante que la televisión, y la posibilidad de encontrar una pulsatila [flor del viento] constituye un derecho tan inalienable como el de libertad de expresión" (Leopold, 1949, Foreword [IX]). En otras palabras, al conocer más y más la naturaleza, se puede aprender de ella a ser más humanos respetándola, siendo así también más naturales. El esfuerzo contemporáneo por comprender, evitar o reducir el impacto de las infraestructuras ligadas al consumo (Heidenreich, 2008) o de las decisiones sobre el medio - natural y social- lo procura y manifiesta. Pero lo muestran también las riquezas para el espíritu humano que grandes ambientalistas han obtenido de su conocimiento y contacto con el mundo natural, entrelazadas en su personalidad científica: «Hay algo infinitamente reparador en los reiterados estribillos de la

2. Texto original: "There are two spiritual dangers in not owning a farm. One is the danger of supposing that breakfast comes from the grocery, and the other that heat comes from the furnace". De traducción no inmediata, el término "spiritual" deba entenderse posiblemente bajo la acepción en su lengua original de "relating to or affecting the human spirit or soul as opposed to material or physical things" (The Oxford Dictionary of English). 
naturaleza, la garantía de que el amanecer viene tras la noche, y la primavera tras el invierno" (Carson, 1956, 45). Entre las personalidades que han considerado la naturaleza como una experiencia espiritual, Caduto cita explícitamente además a Ralph Waldo Emerson, Navarre Scott Momaday, John Muir, el Jefe Seattle, Hildegarda de Bingen, Alce Negro, Lao Tsé, Gibran Jalil Gibran o Francisco de Asís (Caduto, 1998).

\subsection{Sobre las dimensiones materiales y espirituales del medio, su valor y las exigencias a la conducta, y su efecto en individuos específicos}

Con su existencia, la naturaleza ofrece un lenguaje multidimensional que transmite sus secretos propios, eficazmente desvelados por la ciencia, y mensajes específicamente humanos, que van más allá de la materialidad ambiental aunque nazcan con ella.

Aquellos que moran, tanto científicos como profanos, entre las bellezas y misterios de la tierra, nunca están solos o hastiados de la vida. Cualesquiera que sean las contrariedades o preocupaciones de sus vidas, sus pensamientos pueden encontrar el camino que lleve a la alegría interior y a un renovado entusiasmo por vivir. Aquellos que contemplan la belleza de la tierra encuentran reservas de fuerza que durarán hasta que la vida termine (Carson, 1956, 44).

La capacidad de extraer mensajes humanos del mundo natural como los expresados por Carson no es inmediata, ni fácil de poner en práctica. Pero es real. Y, posiblemente, indispensable. Sin necesidad de llegar a las dimensiones que aprehende la poesía (Knickerbocker, 2012), o siguiendo la senda mostrada ocasionalmente en el teatro de Shakespeare ${ }^{3}$, nos exige hacer propio el conocimiento ambiental objetivo y adaptar nuestra conducta a su descubrimiento. No es fácil educarse en la escucha de ese lenguaje de la existencia que acompaña al de la ciencia y solicita el compromiso con el medio ambiente. Los intereses del ser humano pueden ser muy sordos a las existencias que lo rodean, sobre todo si éstas no se quejan en voz alta o amenazante. "Maltratamos a la tierra porque la vemos como una mercancía que nos pertenece" (Leopold, 1949, Foreword [x]). Y también cuando el acceso más profundo al ser de lo natural, acaso el que más compromiso pide a la conducta, la contemplación que sabe ir de la mano tanto de la ciencia experimental como del espíritu, se ha convertido en una destreza humana ignorada, ninguneada, maltratada por las deformidades culturales que se han desarrollado cuando espíritu y ciencia no han querido darse la mano.

3. "And this our life, exempt from public haunt, Finds tongues in trees, books in the running brooks, Sermons in stones, and good in everything", en As you like it, Act. II, Sc.1, de William Shakespeare. 
La pérdida del ejercicio contemplador y del cultivo del espíritu humano individual ante la naturaleza dificulta que descubramos que, además de los mensajes de validez universal que encierra el medio ambiente, éste puede alimentar en personas concretas iniciativas individuales de inmenso valor a su favor, como atestiguan la vida y los logros de los grandes ambientalistas. "Cuando veamos la tierra como una comunidad a la que pertenecemos, tal vez empecemos a usarla con amor y respeto" (Leopold, 1949, Foreword [x]). Esta cuestión, la pertenencia a la tierra, se tratará más adelante. Pero, llegados a este punto, se pasa antes a analizar nuestra relación con el entorno desde un enfoque más negativo.

\subsection{Sobre el vínculo entre el daño ambiental y el daño bumano, material y espiritual, y su reversión a través de la cultura}

Cuando la conducta y elecciones no se adaptan al ser de lo natural y sustraen valor al medio, dañándolo, el ser humano se resta valor junto con él. Sufre entonces las consecuencias que provoca, tanto en la salud (Galea y Vlahov, 2005) como en otros órdenes apuntados por obras como Last Child in the Woods (Louv, 2005). Incluso cuando no es fácil ver el alcance de la dimensión ambiental de nuestra vida y de nuestras elecciones, la conducta en lo referido al medio ambiente no deja de construir o destruir al ser humano y su entorno. Esto sucede haya o no consciencia de lo que ocurre y de sus causas - por ejemplo, a través del comportamiento ambiental de las empresas (Liu y Wu, 2009)-, de forma tanto personal como colectiva -en parte porque el comportamiento ambiental individual no es ajeno al del grupo en que cada persona se integra (Toner, Gan y Leary, 2012)-, y en dimensiones materiales y espirituales (Chuengsatiansup, 2003), en el individuo humano y en su paisaje, con toda la complejidad estructural y funcional que encierra este concepto en la ecología (Verburg, Asselen, Zanden y Stehfest, 2012), desde que Carl Troll lo vinculara a ella de manera formal en 1939. La conducta ambiental que se escoja afecta a todo el ser humano y su expresión territorial y social.

La percepción y reducción del deterioro ambiental va siempre atrasada respecto al daño causado (por ejemplo, la primera Ley de Evaluación Ambiental moderna nace en 1969 en Estados Unidos ${ }^{4}$; en Europa, en 1985'). Y tiende a centrarse en lo más evidente. Se acaba de señalar que la relación que se establece de hecho con el medio ambiente - sea atenta a su cuidado, sea dándole la espalda, más o menos conscientemente- afecta inexorablemente a cada individuo y a la sociedad. Se quiere ahora resaltar que lo hace de modos muy diversos, difíciles de percibir o controlar por completo, y que alcanzan más allá de lo material, como expresan de forma intuitiva estas líneas ya clásicas sobre la relación entre la cultura

4. NEPA, National Environmental Policy Act.

5. Directiva $85 / 337 /$ CEE. 
económica preponderante y su efecto en la salud, aunque su autor no concrete en ellas en qué sentido entenderla: "Nuestra mayor y mejor sociedad es ahora hipocondríaca: está hasta tal punto obsesionada con su salud económica que ha perdido la capacidad de conservar la salud. [...]. Llegados a este punto, nada sería más saludable que un sano desdén por toda una plétora de bienes materiales" (Leopold, 1949, Foreword [xI]).

Ante la libertad de conducta, el medio ambiente natural ofrece un contrapunto, una vía -no la única, por supuesto- para comprender nuestro ser, examinar el comportamiento ambiental y dotarnos de más coherencia conjuntamente natural y humana. Ofrece la oportunidad de dirigirnos con inteligencia y sensibilidad hacia una mayor humanidad, respetándolo por elección. Este ofrecimiento pide, como respuesta, promover una apertura y respeto crecientes hacia lo natural, de forma acompasada con nuestro desarrollo humano personal y colectivo. Se ha escrito que antes de la revolución industrial, momento a partir del cual se acelera el deterioro del medio, el ser humano mantenía una relación relativamente simbiótica con el medio natural, que cambió entonces hacia un consumo rápido y en grandes cantidades de los recursos naturales, sin pensar en su reposición (Olsen y Galimidi, 2009). Acaso se deba, por tanto, ir desandando caminos culturales errados y reaprender actitudes ambientales que parecen olvidadas, más o menos relegadas en la práctica o en la cultura popular contemporáneas. Se trataría de empezar por comprender, aprendiendo a mirar más al fondo de lo natural que en la sola dirección de nuestra conveniencia material inmediata, y así ir alimentando conductas ambientales más acertadas. Las iniciativas legales que buscan la reducción del impacto ambiental -que tiene de por sí un innegable potencial educativo, al mostrar las consecuencias de la acción humana en el medio- van en esa dirección. Son una necesidad cultural y educativa, aunque se piense que llegan tarde o se quedan cortas.

\subsection{Sobre la pertenencia bumana al medio ambiente natural}

La pertenencia humana al medio es vivida en variedad de grados y expresiones por todas las personas con sensibilidad ambiental, de forma particular por culturas muy vinculadas a la tierra (Whit, Roberts, Norman y Grieves, 2001) o por los ambientalistas más destacados, como manifiesta la visión de El Hombre y la Tierra que debemos a Félix Rodríguez de la Fuente. Esta pertenencia debe jugar un papel de primer orden en la educación, no sólo la ambiental. Y, sin embargo, la humanidad con la que el ser humano pertenece corporalmente al medio ambiente natural es frecuentemente ignorada. Esta circunstancia invita a redescubrir de continuo el valor -material, humano- del medio ambiente, que permanece aunque se ignore o desprecie.

El ser humano pertenece también a una comunidad material de vida, a un ecosistema, un paisaje -la casa de la vida-, que se encuentran estrechamente integrados en su ser, junto con sus dimensiones no materiales. "La sonoridad armoniosa de los paisajes vivos, es la forma más segura para comprender hasta qué punto 
somos eso que tenemos delante" (Domínguez, 2001, 427). El ecosistema material se hace presente en el yo y en el nosotros de cada cuerpo individual de quien vive en sociedad. El cuerpo atestigua que nuestro ser no es sólo el de individuos sociales, sino también ecosistémicos.

Pero ¿cómo ser más conscientes de la pertenencia humana al medio de modo que se promueva una mayor responsabilidad hacia el mundo natural, respetándolo en las decisiones concretas? La ciencia ecológica ofrece una primera clave, un punto de arranque ineludible. Si antes se destacaba la afirmación de Leopold de que "Cuando veamos la tierra como una comunidad a la que pertenecemos, tal vez empecemos a usarla con amor y respeto", conviene ahora completarla:

No hay otro modo de que la tierra sobreviva al impacto del hombre mecanizado, ni de que podamos cosechar de ella el valor estético con el que puede contribuir a la cultura, a través de la ciencia (Leopold, 1949, Foreword [x]).

El ser humano es corporal y, a la vez, puede escoger su conducta como ningún otro ser. Para que su conducta escogida sea propiamente humana, las elecciones deben ser coherentes con el modo de ser del cuerpo que le constituye. Pero el cuerpo humano no está aislado, como revela particularmente la ecología. El cuerpo integra al ser humano en la naturaleza de un modo particularmente complejo, profundo y difícil de ignorar, siempre que se repare en él y en su carácter relacional. Por tanto, somos seres relacionales, tanto en lo social como en lo referido al medio ambiente natural, y de forma interdependiente. No es de extrañar, a causa de esa interdependencia, que el auge social del individualismo consumista -lejos de defender el valor y los derechos de cada individuo en sociedad de forma respetuosa con su medio- esté acompañado de la desconexión respecto a la naturaleza y del incremento del impacto ambiental de la sociedad de consumo en su conjunto (Koh y Lee, 2012).

Nos enfrentamos al reto, por tanto, de aprender e integrar en nuestra conducta individual y social, en todas y cada una de las disciplinas y ocupaciones humanas, las lecciones que el contacto con el medio natural, las ciencias del medio ambiente, y en particular la ecología, nos ofrecen. Las necesitamos para entendernos a nosotros mismos, de una forma que nos permita construir humanidad en cada lugar social concreto, de la mano de la naturaleza. De esa comprensión del ser humano como perteneciente al medio ambiente natural, necesitado de su aprendizaje continuo -acaso más difícil a medida que las formas de vida urbanas se alejan del contacto con lo natural- depende que "seamos" más y más humanos a través de nuestras elecciones, más y más coherentes con la humanidad de nuestra dimensión corporal. Una dimensión que nos abre, de suyo, a la relación con el ecosistema y a estar necesitados de él como seres humanos, no sólo como cuerpos.

Además de estar vinculado al ecosistema, el cuerpo es algo propio de toda persona humana. Esta doble pertenencia de la corporalidad, al ecosistema y al yo, permite entender mejor la íntima importancia del ecosistema para avanzar en la exploración de la identidad del ser humano. En cualquier caso, la conducta humana 
puede verse muy influida por un modo de entenderse -o de no entenderse en absoluto- en relación con el medio natural. En la medida en la que el valor del medio natural y la influencia que debería alcanzar en la conducta se desconectan -una desconexión que el vínculo en el yo entre el cuerpo humano y la capacidad de elegir la conducta sugiere que es falsa e injusta-, el medio natural se ve más desprotegido -si cabe- frente a los errores prácticos, excesos o delitos ambientales. Esta desconexión facilita que se actúe como si el medio ambiente natural y el ser humano fueran demasiado indiferentes respectivamente. Y así, ignorar que el cuerpo señala una íntima pertenencia al ecosistema, conduce, fácilmente, a negar de hecho que el valor del medio ambiente natural tenga que ser respetado en la conducta, para modelarla.

La doble dimensión de corporeidad y personalidad libre de cada hombre o mujer demanda que la relación con el medio vaya más allá del uso o consumo que acompañan inevitablemente la condición material. La necesidad de entender a cada persona -como tal- contando con su corporeidad -el medio natural en cada yo- empuja a descubrir el valor de la naturaleza no sólo como si fuera un mero material. Al reparar en que el cuerpo del ser humano libre necesita la relación con el ecosistema que lo mantiene, se comprende que el valor del medio ambiente natural, aun cuando no forme parte de nuestros cuerpos, va mucho más allá de su condición de materia a secas, de bien de producción o consumo. No es, por tanto, externo al círculo que contiene lo que calificamos como humano, aunque no forme parte de nuestro cuerpo. Lo indica, por ejemplo, la compatibilidad -hasta molecular- con todo aquello de lo que nos alimentamos, tomado en última instancia del mismo suelo por las plantas y transformado por ellas.

Esta contundencia ecológica del cuerpo apremia a que se vea su vínculo con aquellas otras dimensiones no corporales, propias y exclusivas del ser humano, que deberían subrayar y fundamentar el respeto ambiental, en lugar de ser empleadas para justificar o ejecutar su despojo. El medio ambiente natural no es un hombre o una mujer; pero se propone aquí que se entienda como humano. Al decir que el medio ambiente es humano llegamos al reverso necesario e inseparable de nuestra afirmación de pertenencia a la tierra. Se da así una vuelta de tuerca más a la magistral formulación de Leopold sobre nuestra pertenencia a la comunidad de la tierra. Se facilita con este nuevo paso la comprensión de nuestro ser, llamado a reconstruir la armonía entre naturaleza y cultura. Una tarea más necesaria cuanto más negada es con los hechos. Entonces ¿cuál es el significado de la humanidad del medio ambiente?

\subsection{Sobre en qué sentido el medio ambiente puede ser calificado como bumano, y si debe ser aprendido como tal}

¿Hasta qué punto la pertenencia humana a la tierra exige una determinada conducta ambiental? Por lo general, se aplica el adjetivo bumano a productos del trabajo o culturales. Pero no se designa así al medio natural, aunque sea la 
base de la subsistencia y posibilite la generación de productos. Por esta razón, el medio natural no puede ser considerado humano en el mismo sentido en el que lo son el ser humano o sus productos, también esencialmente distintos entre sí a su vez. Hay que aceptar la analogía del término en su uso cotidiano. Una vez aceptada, ¿tiene sentido que sea mucho más fácilmente considerado bumano lo que procede de la actividad transformadora o creativa de la mujer y el hombre, que la naturaleza que la hace materialmente posible y sustenta su existencia personal? Sin duda, pues la libertad ejercida distingue a los seres humanos de una forma muy particular. Pero entre la fácilmente admitida humanidad de los productos humanos y la más difícilmente admitida humanidad del medio natural, puede asomar algún olvido.

Al empezar este artículo se distinguía entre dos aspectos simultáneos de la relación del ser humano con el medio ambiente: su anclaje necesario en el mundo natural, y sus elecciones que inciden en los aspectos mudables del medio. Ante el asombroso progresar de las ciencias experimentales, que acerca a conocer las leyes necesarias de la naturaleza, no se ha sabido o querido responder con un ritmo similar en el desarrollo de la ética y la conciencia ambiental. Se ha olvidado cultivarlas para que guíen debidamente las elecciones humanas. La falta de una mejor enseñanza ética durante la etapa universitaria, señalada por científicos estadounidenses en ejercicio como la máxima prioridad a atender, y en especial en lo referido a las relaciones con el medio ambiente (Leopold, 2004), es buena muestra de todo el posible alcance de este fenómeno. Y origina y expresa, entre otros efectos, el extrañamiento respecto a la naturaleza en el que anda nuestra cultura. ¿Cómo corregir o paliar ese defecto?

Con el uso del adjetivo bumano, aplicado al medio natural, se propone un modo de entender la realidad que exige una estrategia educativa acorde. Pero ese uso no carece de fundamento. No deriva sólo del loable fin de proteger lo natural, como si le otorgáramos un rango que en realidad no le corresponde. Se propone, en cambio, enseñar que el medio natural es bumano para subrayar que no se puede descubrir y ejercitar todo el valor del ser humano si se prescinde de alguna parte significativa de nuestra relación con el medio natural y nuestra pertenencia a él, o si se desvinculan de su contenido ético. Y esta reflexión afecta al modo de educar.

La asignación del calificativo de bumano al medio natural puede acarrear dos peligros a evitar en la educación, que derivarían de un exceso de identificación entre ambos términos. El primero consistiría en perder el sentido de lo natural, y dejarlo así sometido exclusivamente al interés humano, error con el que estamos muy familiarizados y que deja al medio natural indefenso frente a nuestros excesos. El peligro inverso consistiría en perder el sentido de lo humano, convirtiéndolo en algo indiferenciado del mundo natural. Se daría paso así a una absolutización de lo natural que podría dejar a la persona humana indefensa frente a quien se erigiera en intérprete oficial y exclusivo de las demandas de la naturaleza. No conviene bajar la guardia ante la amenaza del totalitarismo, que podría venir incluso 
disfrazado de medio ambiente, posibilidad que las leyes a favor del medio natural del nazismo dejaron ya entrever (Ramos, 1993). Si en el primer caso se degrada lo natural a mero material, en el segundo se pierde de vista el valor específico del ser humano individual, que trasciende al que le otorga su pertenencia a una comunidad, sea la comunidad social, sea la comunidad de la tierra. Prevenidos ante estos dos posibles peligros, toca cerrar la reflexión por la que se estima pertinente hablar, en la educación, de la naturaleza como algo humano.

La diferencia esencial entre los seres humanos y su medio natural permite -y debe- alimentar el respeto por ambos a través de la importancia y valor del recíproco. Por ser miembros de una comunidad diversa que comparten, el valor de cada ser humano como ser humano debe alimentar el valor del medio natural como medio natural, y viceversa. Si se resta valor a lo natural con la conducta escogida (por ejemplo, usándolo como un mero material) se pierden valores humanos; y si de antemano no se reconoce el valor humano de lo natural (por ejemplo, viendo como alimenta el cuerpo y el espíritu humanos) es más fácil que el valor de la naturaleza no sea respetado. Existe entre ser humano y naturaleza un vínculo -que edifica la comunidad natural y humana a la que pertenecemosque no puede ser obviado sin erosionar el valor de ambos a la vez. Lo humano está en juego en el respeto ético al medio natural, por distintos que sean. El auge del individualismo, sin embargo, dificulta que se perciban los valores de las comunidades a las que pertenecemos, y que se perciban como propias. Las comunidades naturales y humanas no disuelven el valor de los individuos, no eliminan su valor como tales, pues se edifican $-\mathrm{O}$ no- sobre ellos y su conducta. Por el contrario, los llevan a expresiones humanas que no podrían alcanzar como meros individuos.

En ese sentido de complementariedad entre la ecología de la naturaleza y la ética humana se dice que el ser humano es natural y que la naturaleza es humana, sin identificar ambas realidades. Son distintas, pero su valor es recíprocamente alimentado. Al educarnos así se alimenta la convicción de que la naturaleza exige de suyo -y por lo tanto necesita- la protección que merece en cuanto que entra en el ámbito de lo humano. Y la de que el medio natural enseña al ser humano una parte irrenunciable -que se podría caracterizar como transversal a todo el resto de sus dimensiones- de en qué consiste comportarse humanamente. Además, lo posibilita, aunque sólo sea al dar un marco al ejercicio libre de las actuaciones humanas que lo respetan. Ni la arquitectura o el urbanismo, ni el derecho o la ingeniería, ni la agricultura o la pesca, ni el transporte o el comercio, la cultura o la educación pueden desarrollarse con plenitud humana si no incorporan e integran desde sus ámbitos específicos las lecciones que, para cada disciplina que quiera escuchar, el medio ambiente imparte a la conducta. Es en este sentido, entre otros que no se quieren excluir, en el que se propone hablar de la humanidad de lo natural en la educación. O, al menos, explorar esa vía. 


\subsection{Sobre la necesidad de lograr todas las alianzas ambientales posibles}

La intuición de una cierta humanidad del mundo natural ha sido usada interesadamente como derecho de propiedad sin valores naturales a respetar. Muchos factores han podido contribuir a ese fenómeno, cuyos ecos se pueden encontrar en los más variados textos: "Esclavo de la Naturaleza, mientras no tuvo en ella más que la sensación, tórnase el Hombre legislador de la Naturaleza tan pronto como llega a pensarla. La que antes era su dueña y le dominaba con su fuerza, es ahora el objeto de su mirada reguladora. Lo que para el hombre llega a ser objeto, no tiene ya fuerza sobre él; porque el objeto, para ser objeto, ha de sentir la fuerza y poderío del sujeto" (Schiller, 1795, 133). Hasta las vidas de personas o culturas que se han considerado religiosas han asumido negaciones prácticas, en torno al valor de lo natural, en diversas etapas de la historia. La religión misma ha sido sentada en ocasiones en el banquillo de los acusados, en nombre del medio ambiente (Ramos, 1993).

Para ser honestos, habría que señalar también la contribución que han tenido las religiones a través de los siglos a la hora de reconocer y respetar la naturaleza. Para innumerables creyentes de muy variados credos esa naturaleza ha sido a lo largo de siglos o milenios un libro en el que leer a la divinidad, buscarla y acercarse a ella mediante las actitudes básicas de amor que un ser humano requiere para lograrlo en lo posible, también a través de lo material: humildad, respeto y adoración, gratitud y reconocimiento ante los dones recibidos, austeridad en su uso... Todas ellas reñidas con la tendencia a la opulencia y su impacto ambiental. No es de extrañar que desde la Unión Internacional para la Conservación de la Naturaleza (UICN), poco sospechosa de ceder a presiones confesionales, se considere que los líderes religiosos pueden jugar un papel vital en la conservación del medio ambiente, y que cuente en su seno con un Grupo Especialista en Valores Culturales y Espirituales de las Áreas Protegidas trabajando en estas cuestiones, dentro de la Comisión Mundial de Áreas Protegidas (IUCN, 2011; Mallarach, 2008).

Parece, por tanto, que aunque el debate que abrió el famoso artículo de Lynn White no esté cerrado (White, 1967; Ramos, 1993; Minteer, 2005), se está optando, por la vía de los hechos, por contar con el potencial de responsabilidad y respeto ambiental que, desde las ópticas religiosas, puede salir al encuentro de la cultura de respeto que desarrollan, mucho más notoriamente en nuestra sociedad, la ciencia y la sensibilidad ambiental.

Queda mucho trabajo ambiental por hacer para que las actitudes ambientales negligentes o insuficientes, individuales y en sociedad, sean cambiadas. No estaría de más, en bien de la naturaleza, que creyentes y no creyentes pusieran de su parte toda la apertura recíproca que haga falta para facilitar la conversión ambiental, o el cambio de conducta, que cada religión y creyente, que cada sociedad o ciudadano sin credo, que cada cultura, deban emprender siguiendo una ética ambiental que ha sido descuidada. 
Conviene por tanto evitar que se disperse el esfuerzo de quienes, pensando y creyendo de formas diferentes, coinciden sin embargo en su voluntad de promover el cuidado por la tierra. Hace falta poner el valor del medio ambiente al servicio de la lucha que le es propia: la del respeto por el mundo material recibido. Todo esfuerzo que apunte a desvelar ese valor es bienvenido, y necesario. Desde esta perspectiva, no parece casual, ni superfluo, que tantas culturas y religiones tradicionales hablen de la Madre Tierra, con un sentido que unifica tanto lo material como lo espiritual, al que no es nada ajena la Carta de la Tierra. ¿En qué punto de nuestro llamado progreso perdimos esa consciencia de pertenencia, que acude a la relación materno-filial para mostrarse en toda su profundidad? ¿Cabe una imagen - o realidad- más poderosa, al respecto? Habrá que tener muy en cuenta esa visión, para desarrollarla en nuestra educación en la pertenencia a la tierra.

\subsection{Sobre cómo el enfoque espiritual permite afrontar desde cada contexto bumano la escisión entre naturaleza y cultura}

El desarrollo científico, técnico e industrial, y el auge del conocimiento en la sociedad moderna y contemporánea han acontecido de forma paralela al auge del impacto ambiental, el daño a la tierra y el distanciamiento entre naturaleza y cultura. Una distancia, escisión en ocasiones, objeto de reflexión y de brillantes, profundas e inquietantes intuiciones o formulaciones desde el ámbito del pensamiento, como las expresadas por Claudio Magris en El Danubio, al referirse a "la vida que se alimenta de la muerte" (Magris, 1988, 172) o, de una forma más directa, cuando escribe:

La escisión entre naturaleza y cultura produce malestar en esta última. [...] La mundanidad social constituye nuestro único horizonte. El malestar de la civilización, magistralmente evocado por Freud, nace asimismo de una incurable contradicción. La civilización y la moral se basan en una distinción necesaria y difícilmente sustentable, la que existe entre hombres y animales. Es imposible vivir sin destruir la vida animal, aunque sólo sea la de las mínimas existencias que escapan a nuestra percepción, y es imposible reconocer a los animales unos derechos universales e inviolables, considerar desde una óptica kantiana a cada animal como un fin en lugar de un medio; la fraternidad solidaria puede abarcar a toda la humanidad, pero se detiene ahí. Esta imposibilidad hace inevitable la separación entre mundo humano y mundo natural y obliga a la cultura, que lucha contra los sufrimientos que deben soportar los hombres, a construir su edificio sobre el dolor animal, intentando amortiguarlo pero resignándose a no poder eliminarlo. El irredimible dolor de los animales, pueblo oscuro que acompaña como una sombra nuestra existencia, arroja sobre ésta todo el peso del pecado original. La obra de Canetti, especialmente Masa y poder, es el descubrimiento de las tinieblas que se acumulan en nosotros con la muerte de los seres vivos de los que nos alimentamos (Magris, 1988, 147-148). 
Se puede seguir el hilo de estas consideraciones culturales, y centrar la atención en otras escisiones de nuestra cultura y conducta, aparentemente menos inevitables que a las que Magris se refiere. Enorme parece la fractura interna de una inteligencia eficaz, que sin embargo se desarrolla rompiendo la relación con el medio ambiente natural, a través de actitudes y acciones que podemos modificar, aunque parezca difícil: "Como alguien de Alberta, occidental, ¿podía yo ver la naturaleza de otro modo que como un lugar para visitar, disfrutar y después dejar, más bien que como un lugar en el que yo vivía?" (Martin, 2009, 14). ¿Qué es y qué papel juega y debería jugar lo natural en la vida de Occidente, en el resto del mundo? La cuestión, alimentada de forma notable por la emergencia de la ciencia ecológica, sigue motivando investigaciones en múltiples campos. Por ejemplo, con frecuencia se vuelve sobre los pasos de notabilísimos ecólogos como Ramón Margalef, que siguen influyendo en la cultura contemporánea (Homs Ramírez de la Piscina, 2013) o, fuera de España, sobre Rachel Carson, Aldo Leopold, etc. (Goodwin, 2008; Goralnik y Nelson, 2011; Van Horn, 2011). Desde otros ámbitos disciplinarios y geográficos, el neoliberalismo ha sido señalado, particularmente en América Latina, por encarnar esta escisión, que plasma en forma de leyes la división entre naturaleza y cultura, y entre individuos y comunidades (Roa-García, Urteaga-Crovetto y Bustamante-Zenteno, 2013). La escisión entre naturaleza y cultura, por último, está muy presente en el núcleo del problema al que trata de dar repuesta la Carta de la Tierra mediante la idea de comunidad de la vida, entre otras, que se ejerce en la conducta hacia los demás, hacia la Tierra.

Por tanto, la educación y el proceso de crecimiento que cada persona lleva a cabo a lo largo de su vida debe ser integral, abarcar conocimientos, conducta y afectos en los niveles personal, familiar y social... y acontecer en un contexto de respeto por el mundo natural que se exprese no sólo con palabras, sino con hechos materiales concretos desde cada entorno geográfico, laboral, cultural. Cada persona y sociedad están llamadas desde su corporalidad y espíritu a una adecuada conducta ambiental, que esté en diálogo crecientemente coherente con la sensibilidad, inteligencia y libertad humanas. Y así lo viene considerando la educación ambiental desde sus expresiones oficiales más tempranas: la educación ambiental es

... el proceso a través del cual se identifican valores y se aclaran conceptos con el fin de desarrollar las aptitudes y actitudes necesarias para percibir y comprender la interrelación entre el ser humano, su cultura y su entorno biofísico. La educación ambiental también supone el ejercicio de la toma de decisiones y la formulación personal de un código de comportamiento en los temas referentes a la calidad medioambiental (Hesselink y Cervoský, 2008, 7).

En la búsqueda de mayor humanidad a través de una relación justa con el medio ambiente natural, a menudo se olvida que el ser humano también está conformado por la dimensión espiritual a la que se ha aludido desde el principio, y que es necesario desarrollar en particular en la docencia de modo directo. Y 
también de forma transversal, pues la condición corporal (y, por tanto, material, ecológica) posibilita y acompaña inseparablemente los múltiples y diversos empeños humanos que trascienden la materia y ecología que nos constituyen. Y para que la conducta acorde con la ecología que exige la corporalidad no sea desmentida en la práctica, debe ser atendida desde cada campo específico e integrada en él como algo propio por quienes lo desarrollan con su quehacer, sobre todo en sus expresiones ambientales. Así se evita, por ejemplo, que el empeño ecológico se perciba como una imposición exterior por parte de las disciplinas ambientales, o a cargo de agentes sociales o culturales con intereses particulares ("ecologistas", "verdes", "políticos"...), en lugar de ser percibidos como lo que son: una exigencia humana.

El desempeño de quienes no se dedican directamente a proteger la naturaleza, tiene una vertiente material y ecológica, específica de sus profesiones y quehaceres, que se añade a los aspectos ecológicos que tienen en común entre ellas. De cada una de esas vertientes específicas nace la necesidad de despertar el respeto humano (material y espiritual) por el medio natural desde cada disciplina, campo profesional o quehacer humano. Las posibilidades de este enfoque las ilustra, por ejemplo, la actividad literaria de Joan Maragall (1860-1911). Con su poesía La Fageda d'En Jordà causó de forma no buscada, a la vuelta de un siglo, la protección del entorno que la inspiró, que fue declarado reserva integral, dentro del entonces Paraje Natural de Interés Nacional de la Zona Volcánica de la Garrotxa (Mallarach, Comas y De Armas, 2012). Es momento de reconsiderar, por tanto, el título y el intento de este artículo, y continuar con todo el desarrollo precedente.

La ciencia experimental está abierta al descubrimiento de sentido, y lo alimenta. Pero no es ella misma quien lo descubre, sino el espíritu humano, tanto del científico como de quien está abierto a la ciencia sin ejercerla. El "sentido", entendido como razón de ser o finalidad, no es experimentable al modo de las ciencias empíricas. Por más que se pueda descubrir ocasionalmente a través de experiencias significativas personales, que llegan incluso a transformar radicalmente la vida y conducta de quienes las experimentan, y que por este motivo interesan a la educación ambiental (Cachelin, Paisley y Blanchard, 2009; Chawla, 2006; Walker, 2006). Pese a no ser él mismo experimentable, el sentido se puede inferir a partir de hechos experimentables, y en distintos niveles complementarios a la vez, por mecanismos mucho más habituales que las experiencias significativas referidas. Por ejemplo, en una relación trófica, una realidad experimentable, se puede apreciar a la vez no sólo una finalidad cumplida para el que se nutre, sino también, a su través, para el funcionamiento del ecosistema en el que está integrado... y así ascendientemente, hasta llegar acaso a los aspectos culturales más profundos del arte culinario, que van más allá de la ciencia empírica sin ser ajenos a ella. Por razones análogas, en el desarrollo armónico del ser humano tiene mucho que ver la llamada inteligencia espiritual, que ha sido definida más arriba como «... nuestra capacidad humana de plantear preguntas fundamentales sobre el sentido de la vida; y de experimentar simultáneamente la perfecta conexión entre cada uno de 
nosotros y el mundo en el que vivimos" (Wolman, 2001, 1). Esta expresión de la inteligencia permite la comprensión y mejora de nuestro mundo interior a través de la contemplación y comprensión del mundo exterior... y, en cierto modo, también al revés, en consonancia con lo que se ha venido desarrollando a lo largo de todo el artículo.

En este contexto es más fácil comprender por qué justamente se afirma y propone que la educación ambiental fomente la inteligencia espiritual, o que se apoye en ella... y viceversa. Entre las causas que pueden explicar el deterioro del entorno humano se encuentra la desvalorización espiritual del medio ambiente natural. Un medio ambiente que sólo es valorado en función de su utilidad económica, al que se excluye de todo sentido que no sea reductivamente material, es un medio ambiente condenado a un uso sin más criterio que el del mercado y la legalidad vigente. Y ese criterio es demasiado pobre guardián para todo el valor de lo natural y de lo humano. Con tan limitadas ayudas, sin duda, el medio ambiente natural $-\mathrm{y}$ con él la humanidad de nuestra cultura- queda condenado a su erosión y pérdida progresiva: lo vemos día a día en nuestro entorno.

Si se descuida la dimensión espiritual del medio ambiente natural, éste queda más fácilmente reducido a un vacío de sentido para cada yo. Un vacío que ya no exige nada a nuestra conducta. Prospera entonces la invitación colectiva a la rapiña propia de los momentos en los que los valores son silenciados, en los que todo suele ocurrir por la vía de los hechos, evitando preguntas que los juzguen. El vacío así generado es más doloroso, si cabe, al considerar que se da justo en aquellos lugares de la personalidad, del espíritu humano, donde debería abundar y florecer el arraigo ambiental del individuo. Un arraigo que se podría caracterizar tentativamente como esa relación de búsqueda y encuentro vital pleno, consciente, profundo y amante, que responde al sentido de pertenencia recíproca-corporal y espiritual- entre el ser humano y la tierra, que la educación ambiental debe promover.

\section{CONCLUSIONES}

1) El cuerpo humano hace presente en el yo al ecosistema, que es la casa de la vida. El ser humano queda así integrado en la comunidad inseparablemente material y social de la tierra.

2) La dependencia irrenunciable respecto al ecosistema que tiene el cuerpo humano para poder subsistir enseña que el medio ambiente no es una cuestión superficial o reductivamente material, sino capital para entender la identidad humana y personal en sociedad.

3) Es necesario aprender a discernir y coordinar, individual y socialmente, entre el alcance de las reglas que las ciencias experimentales y la ecología desvelan -que demandan un respeto acorde a los valores que señalan-, y el poder de actuar y aprovechar creativamente la naturaleza, respetando 
134 JORDI PUIG BAGUER* ${ }^{*}$, FERNANDO ECHARRI IRIBARREN*** Y MARÍA CASAS JERICÓ**** EDUCACIÓN AMBIENTAL, INTELIGENCIA ESPIRITUAL Y NATURALEZA

y enriqueciendo el mundo natural y humano (como logran, p. ej., la restauración ecológica... o el arte).

4) Las ciencias de la naturaleza y la ecología son indispensables para alcanzar un comportamiento humano. Pero el lenguaje de la naturaleza es multidimensional, y es necesario estar abierto a los mensajes y las exigencias de comportamiento que cada una de sus dimensiones reclama. Todas las disciplinas, profesiones y quehaceres humanos están llamados a responder coordinadamente al reto ambiental atendiendo a lo que revelan las distintas dimensiones ambientales que sus lenguajes respectivos descubren.

5) Además de los mensajes y exigencias de validez universal, el medio natural puede albergar mensajes personales que alimenten iniciativas humanas individuales de inmenso valor a favor del medio ambiente comunitario, como atestigua la vida de los grandes ambientalistas. Por lo tanto, pese a que no sea fácil educar en su escucha, no se puede pasar por alto este potencial en la educación.

6) A causa del vínculo entre el ser humano y el medio ambiente, el daño ambiental, acelerado particularmente a partir de la revolución industrial, repercute inevitablemente en el propio ser humano. Lo hace individual y socialmente, a través de múltiples caminos -materiales o espirituales- que en ocasiones tardan en ser descubiertos o percibidos.

7) Es una tarea para la educación y la cultura el ir desandando la desconexión o escisión consolidadas entre el medio ambiente natural y el ser humano, resaltados frecuentemente por el fenómeno de los impactos ambientales negativos. Al estudiarlos y hacerles frente se expresa y alimenta una importante función educativa y cultural.

8) El cuerpo humano pertenece a cada persona y está integrado en el ecosistema a la vez, de forma distinta pero inseparable. El cuerpo interactúa necesariamente con su ambiente, como revela particularmente la ecología. La unión del cuerpo humano a la naturaleza empuja a descubrir en ella un valor superior al de un mero material. Ignorar que el cuerpo señala una íntima pertenencia humana al ecosistema conduce, fácilmente, a debilitar el respeto por el valor del medio ambiente natural.

9) El ser humano pertenece a la comunidad de la tierra tanto corporalmente como con las dimensiones humanas no corpóreas que permiten respetar la tierra mediante conductas escogidas. Se debe promover la consciencia y el ejercicio de estos dos aspectos inseparables de la pertenencia al medio natural como tales.

10) Las ciencias naturales, y en particular la ecológica, ofrecen un punto de partida ineludible, aunque insuficiente por sí solo, para superar los problemas ambientales. El actuar humano que tiene incidencia en el medio ambiente se despliega según toda la variedad de disciplinas y artes humanos, que son por tanto necesarias y convocadas para buscar 
conjuntamente con la ecología la solución de cada problema ambiental. Una solución que pide aprender a ver la naturaleza como algo más que un mero material, a considerarla contenida en el círculo de lo que se califica como humano, y que reclama el compromiso ético.

11) $\mathrm{Al}$ progreso de las ciencias experimentales, que acerca a conocer las leyes de la naturaleza, no se ha sabido o querido responder con un ritmo similar en el desarrollo de la ética y la conciencia ambiental. Se ha olvidado cultivar debidamente estas últimas, de forma consecuente con las primeras, para que guíen mejor las elecciones humanas que atañen a la naturaleza.

12) Se propone que el medio ambiente sea designado como humano para resaltar que no se puede descubrir y ejercitar todo el valor del ser humano si se prescinde de alguna parte significativa de nuestra relación con el medio natural y nuestra pertenencia a él, o si se desvinculan de su contenido ético. Y esta reflexión afecta al modo de educar, que debe evitar tanto que se pierda el sentido de lo natural, dejándolo expuesto al mero interés humano, como que se pierda el sentido de lo específicamente humano, que podría dejar al ser humano expuesto a la voluntad de otros seres humanos que se atribuyeran en exclusiva la interpretación oficial de las demandas de la naturaleza.

13) El valor del ser humano como ser humano debe alimentar el valor del medio natural como medio natural, y viceversa, por ser ambos términos miembros de una misma comunidad natural y social que comparten, aunque no se identifiquen entre sí en ella. Si se resta valor a lo natural con la conducta escogida se pierden valores humanos; y si de antemano no se reconoce el valor humano de lo natural es más fácil que el valor de la naturaleza no sea respetado.

14) Existe entre ser humano y naturaleza un vínculo que no puede ser alterado sin erosionar el respeto por ambos a la vez. Su valor respectivo es recíprocamente dependiente. Al educarnos así se alimenta la convicción de que la naturaleza exige de suyo -y por lo tanto necesita- la protección que merece en cuanto que entra en el ámbito de lo humano, que debe ser atendida desde la conducta de toda la variedad de profesiones y quehaceres humanos.

15) La espiritualidad no tiene por qué abordarse desde enfoques religiosos específicos. Pero el debate sobre el papel de las religiones en relación al medio ambiente no debe dificultar las alianzas posibles para respetar la naturaleza. Se debe evitar que se disperse el esfuerzo de quienes, pensando y creyendo de formas diferentes, coinciden sin embargo en su voluntad de promover el cuidado por la tierra. Hace falta poner el valor del medio ambiente al servicio de la lucha que le es propia: la del respeto por el mundo, material y espiritual, humano, que hemos recibido. 
16) La inteligencia espiritual permite contrarrestar la escisión entre naturaleza y cultura que se vuelve a la vez contra las dos. Desde el punto de partida ineludible que ofrece la ciencia experimental, la inteligencia espiritual puede ayudar a alcanzar el sentido de lo natural que se escapa a la ciencia experimental, y que es necesario sin embargo para que ésta pueda alcanzar toda la influencia ética que está llamada a desempeñar y reclamar en nuestra conducta cotidiana, desde todas las profesiones y quehaceres como algo propio de ellas. Los enfoques espirituales y la ciencia experimental pueden alimentar recíprocamente su eficacia en la defensa humana del medio ambiente natural.

17) Nos enfrentamos al reto de aprender e integrar de continuo en nuestra conducta individual y social, en todas y cada una de las disciplinas y ocupaciones humanas, las lecciones que el contacto con el medio natural, las ciencias del medio ambiente, y en particular la ecología, nos ofrecen. Las necesitamos para entender nuestra identidad de una forma que nos permita construir humanidad en cada lugar social concreto, de la mano de la naturaleza y su conocimiento. De esa comprensión del ser humano depende que "seamos" más y más humanos a través de nuestras elecciones, más y más coherentes con la humanidad de nuestra dimensión corporal. Una dimensión que nos abre, de suyo, a la relación con el ecosistema y a estar necesitados de él como seres humanos, no sólo como cuerpos.

\section{APLICACIÓN DOCENTE}

Entre las posibles aplicaciones docentes que las conclusiones anteriores pueden alimentar, dos de los autores de este artículo acaban de editar un texto dirigido a escolares de en torno a 12 años, que propone una serie de imágenes del mundo natural, con ocasión de las cuales se plantea una serie de preguntas que buscan estimular en los escolares el ejercicio de la inteligencia espiritual ante lo que ven, en búsqueda de los valores naturales. No se ofrecen respuestas, dejando el papel de guiar las que los alumnos puedan encontrar a sus educadores respectivos.

\section{REFERENCIAS BIBLIOGRÁFICAS}

Baca-Motes, K.; Brown, A.; Gneezy, A.; Keenan, E. y Nelson, L. D. (2013) Commitment and Behavior Change: Evidence from the Field. Journal of Consumer Research, 39 (5), 1070-1084. Consultado el 14 de octubre de 2014. http://www.jstor.org/stable/info/10.1086/667226

BARTON, J. R. (2006) Eco-dependency in Latin America. Singapore Journal of Tropical Geography, 27 (2), 134-149. Consultado el 14 de octubre de 2014. http://doi.wiley.com/10.1111/j.1467-9493.2006.00249.x 
Benson, P. L. y Roehlkepartain, E. C. (2008) Spiritual development: A missing priority in youth development. New Directions for Youth Development, 2008 (118), 13-28. Consultado el 14 de octubre de 2014.

http://onlinelibrary.wiley.com/doi/10.1002/yd.253/pdf

Cachelin, A.; Paisley, K. y Blanchard, A. (2009) Using the Significant Life Experience Framework to Inform Program Evaluation: The Nature Conservancy's Wings \& Water Wetlands Education Program. The Journal of Environmental Education, 40 (2), 2-15. Consultado el 14 de octubre de 2014.

http://www.tandfonline.com/doi/abs/10.3200/JOEE.40.2.2-14\#.VD_wFPl_vTo

Caduto, M. J. (1998) Ecological Education A System Rooted in Diversity. The Journal of Environmental Education, 29 (4), 11-16. Consultado el 14 de octubre de 2014. http://www.tandfonline.com/doi/abs/10.1080/00958969809599123

CHAwla, L. (2006) Research methods to investigate significant life experiences: review and recommendations. Environmental Education Research, 12 (3-4), 359-374. Consultado el 14 de octubre de 2014.

http://www.tandfonline.com/doi/abs/10.1080/13504620600942840

Chuengsatiansup, K. (2003) Spirituality and health: an initial proposal to incorporate spiritual health in health impact assessment. Environmental Impact Assessment Review, 23, 3-15. Consultado el 14 de octubre de 2014.

http://www.sciencedirect.com/science/article/pii/S0195925502000379

Domínguez, J. J. (2001) Recensión de: ARAujo, J. (2000) La naturaleza, nuestro lujo. Barcelona, Plaza y Janés Editores, en Revista de Educación, 324, 426-427. Consultado el 14 de octubre de 2014.

http://www.mecd.gob.es/dctm/revista-de-educacion/articulosre324/re3242409310. pdf?documentId $=0901 \mathrm{e} 72 \mathrm{~b} 8125 \mathrm{df} 62$

ERnst, A. y Wenzel, U. (2014) Bringing Environmental Psychology Into Action. European Psychologist, 19 (2), 118-126. Consultado el 14 de octubre de 2014.

http://psycontent.metapress.com/openurl.asp?genre=article\&id=doi:10.1027/1016-9040 /a000174

http://dx.doi.org/10.1027/1016-9040/a000174

Friedman, T. L. (2008) Hot, Flat, and Crowded: Why We Need a Green Revolution and How It Can Renew America. New York, Farrar, Straus and Giroux.

Galea, S. y Vlahov, D. (eds.) (2005) Handbook of Urban Health. Populations, Methods and Practice. New York, Springer.

http://dx.doi.org/10.1007/b104167

Gardner, H. (1983) Frames of Mind: The Theory of Multiple Intelligences. New York, Basic Books.

GaRdner, H. (2000) Intelligence Reframed: Multiple Intelligences for the 21st Century. New York, Basic Books.

GÄrLING, T. (2014) Past and Present Environmental Psychology. European Psychologist, 19 (2), 127-131. Consultado el 14 de octubre de 2014.

http://psycontent.metapress.com/content/wv83286246u35352/?genre=article\&id $=$ doi $\%$ $3 \mathrm{a} 10.1027 \% 2 \mathrm{f} 1016-9040 \% 2 \mathrm{fa} 000184$

Goodwin, C. D. (2008) Ecologist Meets Economics: Aldo Leopold, 1887-1948. Journal of the History of Economic Thought, 30 (04), 429-452. Consultado el 14 de octubre de 2014. http://www.journals.cambridge.org/abstract_S1053837207000429 
Goralnik, L. y Nelson, M. P. (2011) Framing a Philosophy of Environmental Action: Aldo Leopold, John Muir and the Importance of Community. The Journal of Environmental Education, 42 (3), 181-192. Consultado el 14 de octubre de 2014. http://www.tandfonline.com/doi/abs/10.1080/00958964.2010.526152

Hamilton, C. (2010) Consumerism, self-creation and prospects for a new ecological consciousness. Journal of Cleaner Production, 18 (6), 571-575. Consultado el 14 de octubre de 2014 . http://linkinghub.elsevier.com/retrieve/pii/S0959652609003023

HART, P. (2010) No Longer a "Little Added Frill": The Transformative Potential of Environmental Education for Educational Change. Teacher Education Quarterly, 37 (4), 155-177. Consultado el 14 de octubre de 2014. http://eric.ed.gov/?id=EJ904906

Hedlund-De WitT, A. (2013) Pathways to Environmental Responsability: A Qualitative Exploration of the Spiritual Dimension of Nature Experience. Journal for the Study of Religion, Nature and Culture, 72 (2), 154-186. Consultado el 14 de octubre de 2014. http://www.equinoxpub.com/journals/index.php/JSRNC/article/view/14971

HeidenReich, E. (2008) Spaces of flow as technical and cultural mediators between society and nature. Environment, Development and Sustainability, 11 (6), 1145-1154. Consultado el 14 de octubre de 2014. http://link.springer.com/10.1007/s10668-008-9171-2

Hesselink, F. y CERVOSKÝ, J. (2008) Learning to Change the Future. A bird's-eye of the history of the IUCN Commision on Education and Communication. Switzerland, IUCN-CEC. Consultado el 14 de octubre de 2014. http://cmsdata.iucn.org/downloads/cec_history_30sept08_draft.pdf

Homs RAmírez de la PISCINA, P. (2013) El dualisme natura/cultura en ecologia. Anàlisi del pensament ecològic margalefià $i$ de les pràctiques de les cooperatives de consum ecològic. Universitat de Barcelona. Consultado el 14 de octubre de 2014. http://diposit.ub.edu/dspace/handle/2445/35658

Hughes, J. D. (2001) An Environmental History of the World: Humankind's Changing Role in the Community of Life. London, Routledge.

IUCN (2011) Religious leaders can play a vital role in environmental conservation. Consultado el 14 de octubre de 2014.

http://www.iucn.org/news_homepage/news_by_date/?7745/Religious-leaders-canplay-a-vital-role-in-environmental-conservation

Jennings, P. A. (2008) Contemplative education and youth development. New Directions for Youth Development, 2008 (118), 101-105. Consultado el 14 de octubre de 2014. http://www.prevention.psu.edu/projects/documents/jennings2008-newdirections.pdf

KaISER, F. G. (2014) Using Cutting-Edge Psychology to Advance Environmental Conservation. European Psychologist, 19 (2), 81-83. Consultado el 14 de octubre de 2014. http://psycontent.metapress.com/content/dq63333w5618454x/fulltext.pdf

KerRet, D. y Ronen, T. (2014) Green Perspective for a Hopeful Future: Explaining Green Schools' Contribution to Environmental Subjective Well-Being. Review of General Psychology, 18 (2), 82-88. Consultado el 14 de octubre de 2014. http://psycnet.apa.org/journals/gpr/18/2/82/

KNiCKerboCKer, S. (2012) Ecopoetics: The language of Nature, the Nature of Language. Massachusetts, University of Massachusetts Press. 
KoH, L. P. y Lee, T. M. (2012) Sensible consumerism for environmental sustainability. Biological Conservation, 151 (1), 3-6. Consultado el 14 de octubre de 2014. http://linkinghub.elsevier.com/retrieve/pii/S0006320711003983

KoH, L. P. y Lee, T. M. (2012) Sensible consumerism for environmental sustainability. Biological Conservation, 151 (1), 3-6. Consultado el 14 de octubre de 2014. http://linkinghub.elsevier.com/retrieve/pii/S0006320711003983

Leopold, A. (1949) A Sand County Almanac. New York, Oxford University Press.

Leopold, A. (2004) Living with the Land Ethic. Bioscience, 54 (2), 149-154. Consultado el 14 de octubre de 2014.

http://bioscience.oxfordjournals.org/content/54/2/149.full.pdf

LIU, C. Y. y Wu, C. H. (2009) Environmental Consciousness, Reputation and Voluntary Environmental Investment. Australian Economic Papers, 48 (2), 124-137. Consultado el 14 de octubre de 2014.

http://doi.wiley.com/10.1111/j.1467-8454.2009.00366.x

Louv, R. (2005) Last Child in the Woods. New York, Algonquin Books.

MAgris, C. (1988) El Danubio. Barcelona, Editorial Anagrama.

Mallarach, J. M. (2008) Protected Landscapes and Cultural and Spiritual Values (vol. 2). Consultado el 14 de octubre de 2014.

https://portals.iucn.org/library/efiles/documents/2008-055.pdf

Mallarach, J. M.; Comas, E. y De ARMas, A. (2012) El patrimonio inmaterial: valores culturales y espirituales. Manual para su incorporación en las áreas protegidas. Madrid, Fundación Fernando González Bernáldez.

Martin, K. (2009) Nature/Culture Breaks: Re-modelling the desire to escape. Continuum, 23 (1), 13-18. Consultado el 14 de octubre de 2014. http://www.tandfonline.com/doi/abs/10.1080/10304310802618350

MinteER, B. A. (2005) An Appraisal of the Critique of Anthropocentrism and Three Lesser Known Themes in Lynn White's "The Historical Roots of our Ecologic Crisis". Organization E Environment, 18 (2), 163-176. Consultado el 14 de octubre de 2014. http://oae.sagepub.com/content/18/2/163.full.pdf

Morin, E. (2006) Organization and Complexity. Annals of the New York Academy of Sciences, 879 (1), 115-121. Consultado el 14 de octubre de 2014. http://onlinelibrary.wiley.com/doi/10.1111/j.1749-6632.1999.tb10410.x/abstract

Newman, P. (2006) The environmental impact of cities. Environment and Urbanization, 18 (2), 275-295. Consultado el 14 de octubre de 2014.

http://eau.sagepub.com/cgi/doi/10.1177/0956247806069599

Olivos-Jara, P.; Aragonés, J. I. y Navarro-Carrascal, O. (2013) Educación ambiental: itinerario en la naturaleza y su relación con conectividad, preocupaciones ambientales y conducta. Revista Latinoamericana de Psicología, 45 (3), 503-513. Consultado el 14 de octubre de 2014: http://www.redalyc.org/articulo.oa?id=80529820014

Olsen, S. y GALIMIDI, B. (2009) Managing Social and Environmental Impact: A New Discipline for a New Economy. Brown Journal of World Affairs, XV (II), 43-56. Consultado el 14 de octubre de 2014.

http://svtgroup.net/wp-content/uploads/2011/09/Managing-Social-and-Environmental-Impact.pdf

Ramos, Á. (1993) ¿Por qué la conservación de la naturaleza? Madrid, Fundación Conde del Valle de Salazar. 
Roa-García, M. C.; Urteaga-Crovetto, P. y Bustamante-Zenteno, R. (2013) Water laws in the Andes: A promising precedent for challenging neoliberalism. Geoforum. Consultado el 14 de octubre de 2014.

http://linkinghub.elsevier.com/retrieve/pii/S001671851300256X

Rоoт, E. (2010) This Land is Our Land? This Land is Your Land: The Decolonizing Journeys of White Outdoor Environmental Educators. Canadian Journal of Environmental Education, 15, 103-119. Consultado el 14 de octubre de 2014. http://cjee.lakeheadu.ca/index.php/cjee/article/view/858/608

Schelly, C.; Cross, J. E.; Franzen, W.; Hall, P. y Reeve, S. (2012) How to Go Green: Creating a Conservation Culture in a Public High School Through Education, Modeling, and Communication. The Journal of Environmental Education, 43 (3), 143-161. Consultado el 14 de octubre de 2014.

HTTP://WWW.TANDFONLINE.COM/DOI/ABS/10.1080/00958964.2011.631611

SCHILlER, F. (1795) La educación estética del hombre. Madrid, Espasa Calpe.

SlabBerT, J. y HATTingh, A. (2006) "Where is the post-modern truth we have lost in reductionist knowledge?". A curriculum's epitaph. Journal of Curriculum Studies, 38 (6), 701-718. Consultado el 14 de octubre de 2014.

http://www.tandfonline.com/doi/abs/10.1080/00220270600608023

TAYlor, D. E. (ed.) (2010) Environment and Social Justice: An International Perspective. Bingley, Emerald Group Publishing Ltd.

TONER, K.; GAN, M. y LEARY, M. R. (2012) The Impact of Individual and Group Feedback on Environmental Intentions and Self-Beliefs. Environment and Behavior, 46 (1), 24-45. Consultado el 14 de octubre de 2014. http://EAB.SAGEPUB.COM/CGI/DOI/10.1177/0013916512451902

VAN HoRn, G. (2011) The (Religious) Naturalist's Eye: An Introduction to "Aldo Leopold: Ethical and Spiritual Dimensions". Journal for the Study of Religion, Nature and Culture, 5 (4), 397-409. Consultado el 14 de octubre de 2014. http://www.equinoxpub.com/JSRNC/article/view/10673

VerburG, P. H.; Asselen, S.; ZANDEn, E. H. y Stehfest, E. (2012) The representation of landscapes in global scale assessments of environmental change. Landscape Ecology, 28 (6), 1067-1080. Consultado el 14 de octubre.

http://link.springer.com/10.1007/s10980-012-9745-0

WalkeR, K. (2006) Doing research in environmental education: touchstone theory and shaking things up. Environmental Education Research, 12 (3-4), 391-401. Consultado el 14 de octubre de 2014.

http://www.tandfonline.com/doi/abs/10.1080/13504620600799125

Whit, L. A.; RoberTs, M.; Norman, W. y Grieves, V. (2001) Belonging to Land: Indigenous knowledge Systems and the Natural World. Oklahoma City University Law Review, 26 (1), 701-744.

White, L. T. J. (1967) The Historical Roots of Our Ecological Crisis. Science, 155 (3767), 12031207. Consultado el 14 de octubre de 2014.

http://oae.sagepub.com/content/18/2/163.full.pdf

http://dx.doi.org/10.1126/science.155.3767.1203

Wolman, R. (2001) Thinking with your soul: spiritual intelligence and why it matters. New York, Harmony Books. 